\title{
A data fusion method in wireless sensor network based on belief structure
}

\author{
Chengfeng Long ${ }^{1 *}$, Xingxin Liu' ${ }^{1}$, Yakun Yang ${ }^{1}$, Tao Zhang ${ }^{1}$, Siqiao Tan ${ }^{1}$, Kui Fang ${ }^{1}$, Xiaoyong Tang ${ }^{2 *}$ and \\ Gelan Yang ${ }^{3}$
}

\author{
${ }^{*}$ Correspondence: \\ elong@hunau.edu.cn; \\ tang_313@163.com \\ ${ }^{1}$ School of Information \\ and Intelligence Science \\ and Technology, Hunan \\ Agricultural University, \\ Changsha, China \\ ${ }^{2}$ School of Computer \\ and Communications \\ Engineering, Changsha \\ University of Science \\ and Technology, Changsha, \\ China \\ Full list of author information \\ is available at the end of the \\ article
}

\begin{abstract}
Considering the issue with respect to the high data redundancy and high cost of information collection in wireless sensor nodes, this paper proposes a data fusion method based on belief structure to reduce attribution in multi-granulation rough set. By introducing belief structure, attribute reduction is carried out for multi-granulation rough sets. From the view of granular computing, this paper studies the evidential characteristics of incomplete multi-granulation ordered information systems. On this basis, the positive region reduction, belief reduction and plausibility reduction are put forward in incomplete multi-granulation ordered information system and analyze the consistency in the same level and transitivity in different levels. The positive region reduction and belief reduction are equivalent, and the positive region reduction and belief reduction are unnecessary and sufficient conditional plausibility reduction in the same level, if the cover structure order of different levels are the same the corresponding equivalent positive region reduction. The algorithm proposed in this paper not only performs three reductions, but also reduces the time complexity largely. The above study fuses the node data which reduces the amount of data that needs to be transmitted and effectively improves the information processing efficiency.
\end{abstract}

Keywords: Wireless sensor network, Granular computing, Rough set, DempsterShafer theory, Reduction

\section{Introduction}

Wireless sensor network (WSN) is a multi-hop self-organizing system formed by wireless sensor nodes communicating with each other and connects the logic world with physical world, which have been considered as one of the most potential and influential technologies. However, due to the short communication distance of sensor nodes, limited information processing capabilities and the inability of a single node to provide all information, sensor nodes can only be deployed in an overlapping manner to obtain complete information about the object within the monitoring range. But this will lead to a series of problems such as high circuit complexity, high data redundancy and high node energy consumption. To effectively solve the above problems, this paper studies rough set theory and evidence theory, which can be used for data analysis and provide author(s) and the source, provide a link to the Creative Commons licence, and indicate if changes were made. The images or other third party material in this article are included in the article's Creative Commons licence, unless indicated otherwise in a credit line to the material. If material is not included in the article's Creative Commons licence and your intended use is not permitted by statutory regulation or exceeds the permitted use, you will need to obtain permission directly from the copyright holder. To view a copy of this licence, visit http:// creativecommons.org/licenses/by/4.0/. 
strong support data fusion technology in the real world of imprecise research [1-6] and proposed other two reductions based on the positive reduction. The propose of three reductions will provide novel insights and different angles to information processing in wireless communication and transmission.

The basic structure of rough set theory is an approximation space consisting of a universe of discourse, in which lower and upper approximations are defined to approximate a undefinable set by using equivalence relations [7-9]. Research on rough set mainly focuses on attribute reduction [10] to fuse information on sensor nodes. From the perspective of granular computing, three extensions of rough set model have been proposed in terms of the characters of data, respectively, multi-granulation rough set based on multi-scale, multi-level and multi-angle [11-20]. A general concept of multi-granulation rough set based on multi-scale describes that an attribute of an object can only take one value in a single-scale information system where the object information is reflected at a fixed scale. We call such a single-scale information system as the classic Pawlak's information system. However, in practical, an object could take on as many different hierarchical values under the same attribute with respect to different scales. And, there do exist special relationships among these hierarchical levels. One example is that the examination results of English for students can be recorded as natural numbers between 0 and 100, and it can also be graded as "Excellent," "Good," "Medium," "Bad," "Unacceptable." Sometimes, if needed, it might be graded into two values, "Passed" and "Failed". A hierarchy of such obtained information granules can be organized to a system which is called multi-scale information system.

The evidence theory represents the uncertainty through the belief and plausibility function derived by the mass function which the core concept is belief structure and evidence structure [21-23]. Recently, the combination of evidence theory and rough set model become one of the research hotspots. As introduced in Yao et al. [24], the adequate condition for belief structure exactly exists in the classic rough set. On the above basis, this study was extended to covering rough set by Chen et al. [25, 26], who successfully employ the belief function and the plausibility function to describe the upper and lower approximations of the covering rough set, which means the numerical features of the rough set can be characterized by evidence theory. In particular, from the perspective of information fusion, Lin et al. [27] explore the relationship between evidence theory and classical multi-granulation rough sets, which shows that, in general, the classic optimistic multi-granulation rough set does not have its corresponding belief structure.

By introducing belief structure, this paper firstly studies the evidential characteristics of multi-granulation rough set based on multi-scale. On this basis, the positive region reduction, belief reduction and plausibility reduction are put forward in incomplete multi-granulation ordered information system and then analyze the consistency in the same level and transitivity in different levels, which can reduce data redundancy and circuit complexity and save node limited resources through data fusion.

\section{Method}

This study puts forward the positive region reduction, belief reduction and plausibility reduction in terms of reducing data redundancy of WSN and proposes an algorithm to reduce the time complexity of attribute reduction. This section firstly introduces the 


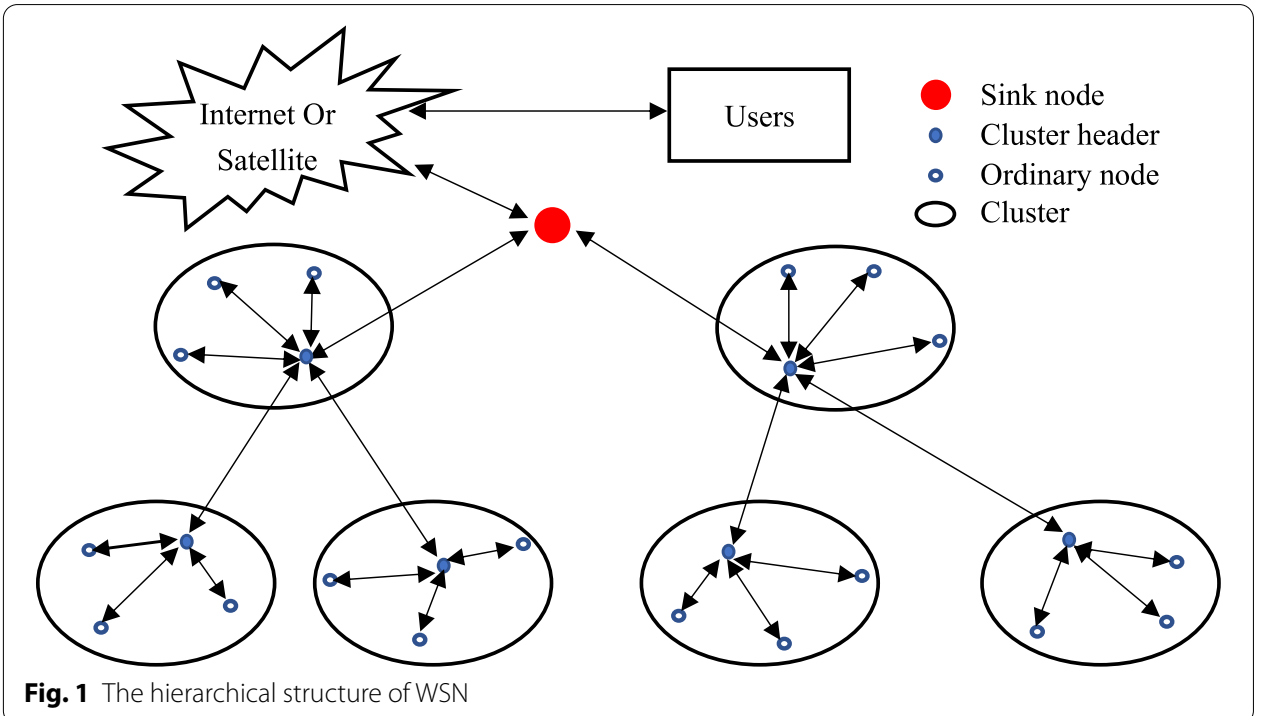

basic preliminaries of WSN information processing, multi-granulation rough set and belief structure. On this basis, the above three reductions are proposed and will be conducted in Sect. 3.

\subsection{Preliminaries}

\subsubsection{WSN information processing}

WSN is mainly composed of nodes, sensor network and users, which the core task of nodes is data perceiving and processing [28-30]. According to a certain standard, $\mathrm{n}$ nodes can form $\mathrm{m}$ clusters and the cluster header is selected in each cluster, which can also represent this cluster at a higher level. Meanwhile, the same mechanism is also applied between cluster headers to form a hierarchical structure [1]. In the above model, the real world is regarded as an information system according to the realistic data observed and measured by WSN. Every single node is considered as an object from the world, and the environment is descripted by a group of attributions which also called observation data. The attribute set can be divided into condition attributes and decision attributes for practical requirements, which are the input and output of the real world, respectively $[1,2,30]$.

This hierarchical routing structure focuses on data which makes the node only interact with their neighbors within a certain range through localized principle, as Fig. 1 shown. And the cluster header will perform data fusion in the cluster so that the sensor node only automatically obtains and transmits effective information. This is also the key to WSN information fusion. In this case, rough set theory particularly suitable for intelligent information fusion at the global level while data from different cluster headers will be aggregate in the sink node, which means that the multi-granulation rough set based on multi-scale can fuse data in the cluster, which ensure that a small amount of effective information is transmitted between the cluster header and sink node with respect to effectively balance information processing, energy consumption and system performance. 


\subsubsection{Multi-granulation rough set based on multi-scale}

Definition 1 [18] Let $U=\left\{x_{1}, x_{2}, \ldots, x_{n}\right\}$ be a nonempty finite set of objects called the universe of discourse, $A T^{k}=\left\{a_{1}^{k}, a_{2}^{k}, \ldots, a_{m}^{k}\right\}$ be a set of attributions and $a_{j}$ be the attribute of multi-granulation. For each object in $U$, the attribute $a_{j}$ can take different values on the different levels of granulations. If $f$ is the attribute value surjective function of different levels (that is, for every $k$ representing the number of levels with a value of positive integer, there exists $x \in U$ such that $f(x)=k)$ and $V^{k}$ is the domain of the attribute $a^{k}$, then the quaternary $M G I S=\left(U, A T^{k}, f^{k}, V^{k}\right)$ is called a multi-granulation information system.

From the above definition, the multi-scale information system will degenerate into the classic Pawlak information system when the number of granular levels is $k=1$. For convenience of description, the following simplifies the multi-granulation information system based on multi-scale as a multi-granulation information system.

Definition 2 [18] Let $M G I S=\left(U, A T^{k}, f^{k}, V^{k}\right)$ be a multi-granulation information system which arbitrary attribute $a_{j}$ has $I$ levels of granulations. We further define the attribute of $a_{j}$ on the $k$-th level of granulations $a_{j}^{k}: U \rightarrow V_{j}^{k}$ represents a surjective function and $V_{j}^{k}$ is the domain of the $k$-th scale attribute $a_{j}^{k}$ (that is, for any $1 \leq k \leq I$, there exists $x \in U$ such that $a_{j}^{k}(x)=*$, where $(*)$ means variable quantity). And the surjective function $g_{j}^{k, k+1}: V_{j}^{k} \rightarrow V_{j}^{k+1}$ (if there exists $k+1$ ) is called the granular transformation function with variable quantity $(*)$ as defined as follows:

$$
a_{j}^{k+1}(x)=\left\{\begin{array}{l}
*, \text { if } a_{j}^{k}=* \\
g_{j}^{k, k+1}\left(a_{j}^{k}(x)\right), \text { if } a_{j}^{k} \neq *
\end{array}\right.
$$

On the basis of Definition 2, clearly, the value of an object between different levels of granulations is not arbitrary and depended on the value of the lower level in a multigranulation information system, which means the value of $a_{j}^{k+1}(x)$ is determined by $a_{j}^{k}(x)$.

Definition 3 [18] Let $M G I S=\left(U, A T^{k}, f^{k}, V^{k}\right)$ be a multi-granulation information system which arbitrary attribute $a_{j}$ has I levels of granulations. For any $1 \leq k \leq I$, the multi-granulation information system MGOIS can be called multi-granulation ordered information system if the attribute value range of any levels of granulations is all partial ordering.

And $M G O I S^{*} \geq$, a multi-granulation ordered information system with variable values $(*)$ and null values, is collectively referred to as an incomplete multi-granulation ordered information system.

Definition 4 [18] Let $M G O I S^{* \geq}=\left(U, A T^{k}, f^{k}, V^{k}\right)$ be an incomplete multi-granulation information system which has $I$ levels of granulations. For any $1 \leq k \leq I$, if the attribute set $A^{k} \subseteq A T^{k}$ and two arbitrary elements $x, y \in U$, then there exists $R_{A T^{k}}^{* \geq}=\left\{(x, y) \in U \times U \mid \forall a^{k} \in A T^{k},\left(f\left(x, a^{k}\right) \leq f\left(y, a^{k}\right)\right) \vee\left(f\left(x, a^{k}\right)=*\right) \vee\left(f\left(y, a^{k}\right)=*\right)\right\}$ 
can be called an incomplete multi-granulation ordered information system dominance relationship.

On the above basis, if the pair $(x, y) \in R_{A T^{k}}^{* \geq}$, then $[X]_{A T^{k}}^{* \geq}$ means that $y$ is finer than $x$ or $x$ is coarser than $y$. The relationship $R_{A T^{k}}^{* \geq}$ can be considered as a kind of surjection from $U$ to $P(U)$ where $P(U)$ is a power set. $U / R=\{y \in P(U) \mid x \in U\}$ is a covering of universe of discourse.

Definition 5 [18] Let $M G O I S^{* \geq}=\left(U, A T^{k}, f^{k}, V^{k}\right)$ be an incomplete multi-granulation ordered information system which has $I$ levels of granulations. For every $X \subseteq U$ and any $1 \leq k \leq I$, the lower and upper approximations of $X$ in the $k$-th level of granulations are defined as $R_{A T^{k}}^{* \geq}(X)=\left\{x \in U \mid[x]_{A T^{k}}^{* \geq} \subseteq U\right\}$ and $\overline{R_{A T^{k}}^{* \geq}}(X)=\left\{x \in U \mid[x]_{A T^{k}}^{* \geq} \cap X \neq \varnothing\right\}$, respectively.

From Definition 5, the relationship between the lower and upper approximations in the same levels of granulations has been clearly proved. $R_{A T^{k}}^{* \geq}(X)$ and $\overline{R_{A T^{k}}^{* \geq}}(X)$ satisfy the following properties, which will be the theoretical foundation for the further discussions in this paper.

Proposition 1 [18] Let $M G O I S^{* \geq}=\left(U, A T^{k}, f^{k}, V^{k}\right)$ be an incomplete multigranulation ordered information system which has I levels of granulations. For any $1 \leq k \leq I$ and two elements $X, Y \subseteq U$, we denote the complement of $X$ in $U$ as $\sim U$, i.e., $\sim U=U-X=\{x \in U \mid x \notin X\}$, then,

$$
\begin{aligned}
& \underline{R_{A T^{k}}^{* \geq}}(X)=\sim \overline{R_{A T^{k}}^{* \pm}}(\sim X) \\
& \overline{R_{A T^{k}}^{* \pm}}(X)=\sim \underline{R_{A T^{k}}^{* \geq}}(\sim X) \\
& \underline{R_{A T^{k}}^{* \pm}}(\varnothing)=\varnothing, \overline{R_{A T^{k}}^{* \pm}}(\varnothing)=\varnothing \\
& \underline{R_{A T^{k}}^{* \pm}}(U)=U, \overline{R_{A T^{k}}^{* \pm}}(U)=U \\
& \underline{R_{A T^{k}}^{* \pm}}(X) \subseteq U \subseteq \overline{R_{A T^{k}}^{* \pm}}(X) \\
& \underline{R_{A T^{k}}^{* \pm}}(X \cap Y)=\underline{R_{A T^{k}}^{* \geq}}(X) \cap \underline{R_{A T^{k}}^{* \pm}}(Y) \\
& \overline{R_{A T^{k}}^{* \pm}}(X \cup Y)=\overline{R_{A T^{k}}^{* \pm}}(X) \cup \overline{R_{A T^{k}}^{* \pm}}(Y)
\end{aligned}
$$

Theorem 1 Let $M G O I S^{* \geq}=\left(U, A T^{k}, f^{k}, V^{k}\right)$ be an incomplete multi-granulation ordered information system which has I levels of granulations. For any $1 \leq k \leq I$ and every $x \in U$, denote attribute subset $A^{k} \subseteq A T^{k}$ then $[x]_{A^{k}}^{* \pm} \subseteq[x]_{A^{k+1}}^{* \pm}$, where $1 \leq k \leq I-1$. 
Proof On the basis of granular transformation function from Definition 2, it is easy to see that for any $a_{j}^{k}$, there exists $g_{j}^{k, k+1}\left(a_{j}^{k}(y)\right) \geq g_{j}^{k, k+1}\left(a_{j}^{k}(x)\right)$ such that $\left\{y \in U \mid \forall a_{j}^{k} \in A^{k}, g_{j}^{k, k+1}\left(a_{j}^{k}(y)\right) \geq g_{j}^{k, k+1}\left(a_{j}^{k}(x)\right)\right\}$. Thus, we have $[x]_{A^{k}}^{* \geq} \subseteq[x]_{A^{K}+1}^{* \geq}$.

Theorem 1 represents the relationship of the attribute set $A T$ in different levels of granulations, i.e., $R_{A T^{k+1}}^{* \geq}$ is subdivided by the relationship $R_{A T^{k}}^{* \geq}$ defined on the attribute set $A T$, and thus obtains the relationship between the upper and lower approximations in different levels of granulations.

Proposition 2 Let $M G O I S^{* \geq}=\left(U, A T^{k}, f^{k}, V^{k}\right)$ be an incomplete multi-granulation ordered information system which has I levels of granulations. For any $1 \leq k \leq I$ and every $x \in U$, denote attribute subset $A^{k} \subseteq A T^{k}$, then,

(1) $R_{A T^{k+1}}^{* \geq}(X) \subseteq R_{A T^{k}}^{* \geq}(X)$ where $1 \leq k \leq I-1$

(2) $\overline{R_{A T^{k}}^{* \geq}}(X) \subseteq \overline{R_{A T^{k+1}}^{* \geq}}(X)$ where $1 \leq k \leq I-1$

Proof

(1) If for every $x \in U$, there exists $x \in R_{A T^{k+1}}^{* \geq}(X)$ such that $[x]_{A T^{k+1}}^{* \geq} \subseteq X$. With theorem 1, if $[x]_{A T^{k}}^{* \geq} \subseteq X$, then $x \in \underline{R}_{A T^{k}}^{* \geq}(X)$. Thus, we have proved that $R_{A T^{k+1}}^{* \geq}(X) \subseteq \underline{R_{A T^{k}}^{* \geq}}(X)$.

(2) If for every $\overline{x \in U}$, there exists $x \in \overline{R_{A T^{k+1}}^{* \geq}}(X)$ such that $[x]_{A T^{k}}^{* \geq} \cap X \neq \varnothing$. With theorem 1, if $[x]_{A T^{k+1}}^{* \geq} \cap X \neq \varnothing$, then $x \in \overline{R_{A T^{k+1}}^{* \geq}}(X)$. Thus, we have proved that $\overline{R_{A T^{k}}^{* \geq}}(X) \subseteq \overline{R_{A T^{k+1}}^{* \geq}}(X)$.

From Proposition 2, clearly, the lower approximation in the $(k+1)$-th level of granulations of $X$ further subdivides the same in the $k$-th and the upper approximation in the $(k+1)$-th level of granulation of $X$ further subdivides the same in the $k$-th. Moreover, we have the corresponding hierarchical sequence of approximations as follows.

$$
\begin{aligned}
& \underline{R_{A T^{k+1}}^{* \geq}}(X) \subseteq \underline{R_{A T^{k}}^{* \geq}}(X) \subseteq \cdots \subseteq \underline{R_{A T^{2}}^{* \geq}}(X) \subseteq \underline{R_{A T^{1}}^{* \geq}}(X) \\
& \overline{R_{A T^{1}}^{* \geq}}(X) \subseteq \overline{R_{A T^{2}}^{* \geq}}(X) \subseteq \cdots \subseteq \overline{R_{A T^{k}}^{* \geq}}(X) \subseteq \overline{R_{A T^{k+1}}^{* \geq}}(X)
\end{aligned}
$$

In the following, employ Example 1 to illustrate $\overline{R_{A T^{1}}^{* \geq}}(X) \subseteq \overline{R_{A T^{2}}^{* \geq}}(X) \subseteq \cdots \subseteq \overline{R_{A T^{k}}^{* \geq}}(X) \subseteq \overline{R_{A T^{k+1}}^{* \geq}}(X)$ for understanding the above eqs more conveniently. Example 1 will provide a table of the quantity of rape pests detected by WSN. For facilitating reduction, in the first level of granulation, the quantity will be regarded as 90 when it is between 80 and 100 or 70 when it is between 60 and 80, and it can deduce the rest from this. However, missing values will exist due to perception errors and access limitations in WSN to a certain extent, and unclear pictures or imprecise targets will be regarded as missing values. 
Table 1 A multi-granulation incomplete ordered information system with three levels of granulations

\begin{tabular}{lllllllllllll}
\hline$U$ & $a_{1}{ }^{1}$ & $a_{1}{ }^{2}$ & $a_{1}{ }^{3}$ & $a_{2}{ }^{1}$ & $a_{2}{ }^{2}$ & $a_{2}{ }^{3}$ & $a_{3}{ }^{1}$ & $a_{3}{ }^{2}$ & $a_{3}{ }^{3}$ & $a_{4}{ }^{1}$ & $a_{4}{ }^{2}$ & $a_{4}{ }^{3}$ \\
\hline$x_{1}$ & 90 & $a$ & $F$ & 90 & $a$ & $F$ & $*$ & $*$ & $*$ & 90 & $a$ & $F$ \\
$x_{2}$ & 70 & $l$ & $F$ & 90 & $a$ & $F$ & 70 & $l$ & $F$ & 90 & $a$ & $F$ \\
$x_{3}$ & $*$ & $*$ & $*$ & 30 & $f$ & $S$ & 10 & $r$ & $S$ & 30 & $f$ & $S$ \\
$x_{4}$ & 50 & $m$ & $F$ & 50 & $m$ & $F$ & $*$ & $*$ & $*$ & 50 & $m$ & $F$ \\
$x_{5}$ & 30 & $f$ & $S$ & 50 & $m$ & $F$ & 30 & $f$ & $S$ & 30 & $f$ & $S$ \\
$x_{6}$ & 70 & $I$ & $F$ & 70 & $I$ & $F$ & 70 & $l$ & $F$ & 70 & $l$ & $F$ \\
$x_{7}$ & 10 & $r$ & $S$ & 30 & $f$ & $S$ & 10 & $r$ & $S$ & 30 & $f$ & $S$ \\
$x_{8}$ & $*$ & $*$ & $*$ & 90 & $a$ & $F$ & 90 & $a$ & $F$ & 90 & $a$ & $F$ \\
$x_{9}$ & 30 & $f$ & $S$ & 50 & $m$ & $F$ & 50 & $m$ & $F$ & 50 & $m$ & $F$ \\
$x_{10}$ & 90 & $a$ & $F$ & $*$ & $*$ & $*$ & 90 & $a$ & $F$ & 90 & $a$ & $F$ \\
$x_{11}$ & 50 & $m$ & $F$ & 70 & $l$ & $F$ & 50 & $m$ & $F$ & 70 & $l$ & $F$ \\
$x_{12}$ & 10 & $r$ & $S$ & 10 & $r$ & $S$ & 10 & $r$ & $S$ & $*$ & $*$ & $*$ \\
\hline
\end{tabular}

Example 1 Table 1 is an incomplete multi-granulation ordered information system table of Rape pests detected by WSN in a certain period of time, where granularity $I=3$, $U=\left\{x_{1}, x_{2}, x_{3}, x_{4}, x_{5}, x_{6}, x_{7}, x_{8}, x_{9}, x_{10}, x_{11}, x_{12}\right\} \quad$ and $\quad A T^{k}=\left\{a_{1}^{k}, a_{2}^{k}, a_{3}^{k}, a_{4}^{k}\right\}$ $X_{i}(i=1,2, \ldots, 12)$ represents different clusters which stand for Cabbage butterfly, Aphids, Cabbage bug and Cricket, respectively. And $A T^{k}$ is the attribute set where $k=1,2,3$, which is the different levels of granulations. And $*$ is the missing value. From a multi-granular information system structure, we obtain the hierarchical sequence of attributes in different levels of granulations as follows.

(1) The sequence of the value in the first level of granulation of the quantity of pests is $\{1<2<\ldots<100\}$.

(2) The sequence of the value in the second level of granulation of the grade of the quantity is $\{r<f<m<l<a\}$ where $r, f, m, l$ and $a$ represent rarely, few medium, lot and abundance, respectively.

(3) The sequence of the value in the third level of granulation of the grade of risk is $\{S<F\}$ where $S$ and $F$ represent Seconds and Firsts, respectively.

For the above levels of granulations, the system is decomposed into three decision tables which are described as Tables 2, 3, 4, respectively.

From Table 2, we can derive that the value of the first level of granulation is as follows.

$$
\begin{aligned}
& {\left[x_{1}\right]_{A T^{1}}^{* \geq}=\left[x_{8}\right]_{A T^{1}}^{* \geq}=\left[x_{10}\right]_{A T^{1}}^{* \geq}=\left\{x_{1}, x_{8}, x_{10}\right\}} \\
& {\left[x_{2}\right]_{A T^{1}}^{* \geq}=\left\{x_{1}, x_{2}, x_{8}, x_{10}\right\}} \\
& {\left[x_{3}\right]_{A T^{1}}^{* \geq}=\left[x_{7}\right]_{A T^{1}}^{* \geq}=\left[x_{12}\right]_{A T^{1}}^{* \geq}=\{U\}}
\end{aligned}
$$


Table 2 The incomplete ordered information table with the first level of granulations

\begin{tabular}{lllll}
\hline$U$ & $a_{1}{ }^{1}$ & $a_{2}{ }^{1}$ & $a_{3}{ }^{1}$ & $a{ }^{1}$ \\
\hline$x_{1}$ & 90 & 90 & $*$ & 90 \\
$x_{2}$ & 70 & 90 & 70 & 90 \\
$x_{3}$ & 30 & 10 & 30 \\
$x_{4}$ & $*$ & 50 & $*$ & 50 \\
$x_{5}$ & 50 & 50 & 30 & 30 \\
$x_{6}$ & 30 & 70 & 70 & 70 \\
$x_{7}$ & 70 & 30 & 10 & 30 \\
$x_{8}$ & 10 & 90 & 90 & 90 \\
$x_{9}$ & $*$ & 50 & 50 & 50 \\
$x_{10}$ & 30 & $*$ & 90 & 90 \\
$x_{11}$ & 90 & 70 & 50 & 70 \\
$x_{12}$ & 50 & 10 & 10 & $*$ \\
\hline
\end{tabular}

Table 3 The incomplete ordered information table with the second level of granulations

\begin{tabular}{lllll}
\hline$U$ & $a_{1}^{2}$ & $a_{2}{ }^{2}$ & $a_{3}{ }^{2}$ & $a_{4}{ }^{2}$ \\
\hline$x_{1}$ & $a$ & $a$ & $*$ & $a$ \\
$x_{2}$ & $l$ & $a$ & $l$ & $a$ \\
$x_{3}$ & $f$ & $r$ & $f$ \\
$x_{4}$ & $*$ & $m$ & $*$ & $m$ \\
$x_{5}$ & $m$ & $m$ & $f$ & $f$ \\
$x_{6}$ & $f$ & $l$ & $l$ & 1 \\
$x_{7}$ & $l$ & $f$ & $r$ & $f$ \\
$x_{8}$ & $r$ & $a$ & $a$ & $a$ \\
$x_{9}$ & $*$ & $m$ & $m$ & $m$ \\
$x_{10}$ & $f$ & $*$ & $a$ & $a$ \\
$x_{11}$ & $a$ & $l$ & $m$ & $l$ \\
$x_{12}$ & $m$ & $r$ & $r$ & $*$ \\
\hline
\end{tabular}

Table 4 The incomplete ordered information table with the third level of granulations

\begin{tabular}{lllll}
\hline$U$ & $a_{1}{ }^{3}$ & $a_{2}{ }^{3}$ & $a_{3}{ }^{3}$ & $a_{4}{ }^{3}$ \\
\hline$x_{1}$ & $F$ & $F$ & $*$ & $F$ \\
$x_{2}$ & $F$ & $F$ & $F$ & $F$ \\
$x_{3}$ & $S$ & $S$ & $S$ \\
$x_{4}$ & $*$ & $F$ & $*$ & $F$ \\
$x_{5}$ & $S$ & $F$ & $S$ & $S$ \\
$x_{6}$ & $S$ & $F$ & $F$ & $F$ \\
$x_{7}$ & $F$ & $S$ & $S$ & $S$ \\
$x_{8}$ & $S$ & $F$ & $F$ & $F$ \\
$x_{9}$ & $*$ & $F$ & $F$ & $F$ \\
$x_{10}$ & $S$ & $*$ & $F$ & $F$ \\
$x_{11}$ & $F$ & $F$ & $S$ & $*$ \\
$x_{12}$ & $F$ & $S$ & $F$
\end{tabular}




$$
\begin{aligned}
& {\left[x_{4}\right]_{A T^{1}}^{* \geq}=\left\{x_{1}, x_{2}, x_{4}, x_{6}, x_{8}, x_{10}, x_{11}\right\}} \\
& {\left[x_{5}\right]_{A T^{1}}^{* \geq}=\left\{x_{1}, x_{2}, x_{4}, x_{5}, x_{6}, x_{8}, x_{9}, x_{10}, x_{11}\right\}} \\
& {\left[x_{6}\right]_{A T^{1}}^{* \geq}=\left\{x_{1}, x_{2}, x_{6}, x_{8}, x_{10}, x_{11}\right\}} \\
& {\left[x_{9}\right]_{A T^{1}}^{* \geq}=\left\{x_{1}, x_{2}, x_{4}, x_{6}, x_{8}, x_{9}, x_{10}, x_{11}\right\}} \\
& {\left[x_{11}\right]_{A T^{1}}^{* \geq}=\left\{x_{1}, x_{2}, x_{6}, x_{8}, x_{10}, x_{11}\right\}}
\end{aligned}
$$

Suppose $X=\left\{x_{1}, x_{6}, x_{8}, x_{10}, x_{11}\right\}$, then the reductions of the lower and upper approximation are $R_{A T^{1}}^{* \geq}(X)=\left\{x_{1}, x_{8}, x_{10}\right\}$ and $\overline{R_{A T^{1}}^{* \geq}}(X)=\{U\}$, respectively.

From Table 3, we can derive that the value of the second level of granulation is as follows.

$$
\begin{aligned}
& {\left[x_{1}\right]_{A T^{2}}^{* \geq}=\left[x_{8}\right]_{A T^{2}}^{* \geq}=\left[x_{10}\right]_{A T^{2}}^{* \geq}=\left\{x_{1}, x_{8}, x_{10}\right\}} \\
& {\left[x_{2}\right]_{A T^{2}}^{* \geq}=\left\{x_{1}, x_{2}, x_{8}, x_{10}\right\}} \\
& {\left[x_{3}\right]_{A T^{2}}^{* \geq}=\left[x_{7}\right]_{A T^{2}}^{* \geq}=\left[x_{12}\right]_{A T^{2}}^{* \geq}=\{U\}} \\
& {\left[x_{4}\right]_{A T^{2}}^{* \geq}=\left\{x_{1}, x_{2}, x_{4}, x_{6}, x_{8}, x_{10}, x_{11}\right\}} \\
& {\left[x_{5}\right]_{A T^{2}}^{* \geq}=\left\{x_{1}, x_{2}, x_{4}, x_{5}, x_{6}, x_{8}, x_{9}, x_{10}, x_{11}\right\}} \\
& {\left[x_{6}\right]_{A T^{2}}^{* \geq}=\left\{x_{1}, x_{2}, x_{6}, x_{8}, x_{10}, x_{11}\right\}} \\
& {\left[x_{9}\right]_{A T^{2}}^{* \geq}=\left\{x_{1}, x_{2}, x_{4}, x_{6}, x_{8}, x_{9}, x_{10}, x_{11}\right\}} \\
& {\left[x_{11}\right]_{A T^{2}}^{* \geq}=\left\{x_{1}, x_{2}, x_{6}, x_{8}, x_{10}, x_{11}\right\}}
\end{aligned}
$$

Suppose $X=\left\{x_{1}, x_{6}, x_{8}, x_{10}, x_{11}\right\}$, then the reductions of the lower and upper approximation are $R_{A T^{2}}^{* \geq}(X)=\left\{x_{1}, x_{8}, x_{10}\right\}$ and $\overline{R_{A T^{2}}^{* \geq}}(X)=\{U\}$, respectively. And for every $x \in U$, we can obtain $[x]_{A T^{1}}^{* \geq} \subseteq[x]_{A T^{2}}^{* \geq}$.

From Table 4, we can obtain that the value of the third level of granulations is as follows.

$$
\begin{aligned}
& {\left[x_{1}\right]_{A T^{3}}^{* \geq}=\left[x_{2}\right]_{A T^{3}}^{* \geq}=\left[x_{4}\right]_{A T^{3}}^{* \geq}=\left[x_{6}\right]_{A T^{3}}^{* \geq}=\left[x_{11}\right]_{A T^{3}}^{* \geq}=\left\{x_{1}, x_{2}, x_{4}, x_{6}, x_{8}, x_{10}, x_{11}\right\}} \\
& {\left[x_{3}\right]_{A T^{3}}^{* \geq}=\left[x_{7}\right]_{A T^{3}}^{* \geq}=\left[x_{12}\right]_{A T^{3}}^{* \geq}=\{U\}}
\end{aligned}
$$




$$
\begin{aligned}
& {\left[x_{5}\right]_{A T^{3}}^{* \geq}=\left\{x_{1}, x_{2}, x_{4}, x_{5}, x_{6}, x_{8}, x_{9}, x_{10}, x_{11}\right\}} \\
& {\left[x_{8}\right]_{A T^{3}}^{* \geq}=\left[x_{9}\right]_{A T^{3}}^{* \geq}=\left[x_{10}\right]_{A T^{3}}^{* \geq}=\left\{x_{1}, x_{2}, x_{4}, x_{6}, x_{8}, x_{9}, x_{10}, x_{11}\right\}}
\end{aligned}
$$

Suppose $X=\left\{x_{1}, x_{6}, x_{8}, x_{1} 0, x_{1} 1\right\}$, then the reduction of the lower and upper approximation are $R_{A T^{3}}^{* \geq}(X)=\varnothing$ and $\overline{R_{A T^{3}}^{* \geq}}(X)=\{U\}$, respectively. And for every $x \in U$, we can obtain $[x]_{A T^{2}}^{* \geq} \subseteq[x]_{A T^{3}}^{* \geq}$

Example 1 illustrates that it is not arbitrary for the value of the same attribute of the same object in different levels of granulations and proves that the value of the higher level of granulations is determined by the lower, i.e., the $a$ from the attribute $a_{1}$ of $x_{1}$ in the second level of granulations is determined by 90 from the value of the first level.

\subsubsection{Evidence structure}

Definition 6 [20-22] Let $\Theta$ be a finite and nonempty set which is called the frame of discernment, where $A$ is the arbitrary subset of $\Theta$. If there exists a mapping function $m: 2^{\Theta} \rightarrow[0,1]$ that satisfies $m(\varnothing)=0$ and $\sum_{X \subseteq U} m(X)=1$, then we define that the function $m$ is the basic probability assignment function or the mass function on $2^{\Theta}$.

The degree of evidence exactly to $A$ is indicated by $m(A)$. If there exists $m(A)>0$, then we suppose that $A$ is called the focal element of $m$ and a family of all focal elements are viewed as the core. A pair of $(F, m)$ is called a belief structure on the core. And we can obtain the other pair of the belief and plausibility functions can be derived as in terms of the mass function as Definition 7.

Definition 7 [20-22] Let $\Theta$ be a finite and nonempty set which is called the frame of discernment, where $A$ is the subset of $\Theta$ and $m$ is the basic probability assignment function of the frame of discernment $\Theta$. The belief function is a mapping $\operatorname{Bel}(X)$ that satisfies $\operatorname{Bel}(X)=\sum_{A \subseteq U} m(A)$ and the plausibility is a mapping $\operatorname{Pl}(X)$ that satisfies $\operatorname{Pl}(X)=\sum_{A \cap X \neq \varnothing} m(A)$.

The Belief function $\operatorname{Bel}(X)$ represents the true degree of trust for $X$, while the plausibility function $P l(X)$ indicates that it is no doubt with trust is not true for $X$. These two functions are based on the same belief structure that are connected by the dual property, i.e., $\operatorname{Bel}(X)=1-\operatorname{Pl}(\sim X)$, where $\sim X$ is the complement of $X$. Also, the belief function can be defined by semi-additive measure as Definition 8 .

Definition 8 [20-22] For the arbitrary subset $2^{\Theta}$ of the frame of discernment, a mapping function $\mathrm{Bel}: 2^{\Theta} \rightarrow[0,1]$ is belief function if it satisfies the following conditions.

(1) $\operatorname{Bel}(\varnothing)=0$

(2) $\operatorname{Bel}(\Theta)=1$

(3) $\operatorname{Bel}\left(\bigcup_{i=1}^{m} X_{i}\right) \geq \sum_{\varphi \neq J(1,2, \ldots, m)}(-1)^{|U+1|} \operatorname{Bel}\left(\bigcap_{i \in J} X_{i}\right)$ 
Theorem 2 Let $M G O I S^{*} \geq=\left(U, A T^{k}, f^{k}, V^{k}\right)$ be an incomplete multi-granulation ordered information system which has I levels of granulations. For any $1 \leq k \leq I$, denote the subset $A^{k} \subseteq A T^{k}$. For every $X \subseteq U$, then, $\operatorname{Bel}_{A^{k}}^{* \geq}(X)=\frac{\left|R_{A^{k}}^{* \geq}(X)\right|}{|U|}$ and $P l_{A^{k}}^{* \geq}(X)=\frac{\left|\overline{R_{A^{k}}^{* \pm}}(X)\right|}{|U|}$ are the belief and plausibility function of the $k$-th level of granulations, respectively, and the corresponding mass function is $m_{A^{k}}(X)=\left\{\frac{\left|\left\{x \in U \mid[x]_{A T^{k}}^{* \geq}=X\right\}\right|}{|U|}\right.$.

Proof According to Definition 6, we can derive that $m_{A^{k}}(X)$ is called mass function and then only need to demonstrate that $\mathrm{Bel}_{A^{k}}^{* \geq}$ satisfies three conditions of Definition 8 . From the basic Definition 7, we take $B e l_{A^{k}}^{* \geq}(\varnothing)=0$ and $B e l_{A^{k}}^{* \geq}(U)=1$, respectively. Next, prove the condition (3) of Definition 8. Considering a collection $\left\{x_{1}, x_{2}, \ldots, x_{n}\right\} \subseteq U$, then we have

$$
\begin{aligned}
& \frac{\left|R_{A^{k}}^{* \geq}\left(X_{1} \cup X_{2} \cup \ldots \cup X_{n}\right)\right|}{|U|} \geq \frac{\left|R_{A^{k}}^{* \geq}\left(X_{1}\right) \cup \underline{R_{A^{k}}^{* \geq}}\left(X_{2}\right) \cup \ldots \cup \underline{R_{A^{k}}^{* \geq}}\left(X_{n}\right)\right|}{|U|} \\
& =\sum_{i} \frac{\left|R_{A^{*}}^{* \geq}\left(X_{i}\right)\right|}{|U|}-\sum_{i<j} \frac{\left|R_{A^{k}}^{* \geq}\left(X_{i}\right) \cap R_{A^{k}}^{* \geq}\left(X_{j}\right)\right|}{|U|}+\ldots \\
& +(-1)^{n+1} \frac{\mid R_{A^{k}}^{* z}\left(X_{1}\right) \cap \frac{R_{A^{k}}^{* \geq}}{\left(X_{2}\right) \cap \ldots \underline{R_{A^{k}}^{* \geq}}\left(X_{n}\right) \mid}}{|U|} \\
& =\sum_{i} \frac{\left|R_{A^{k}}^{* \geq}\left(X_{i}\right)\right|}{|U|}-\sum_{i<j} \frac{\left|R_{A^{*}}^{* \geq}\left(X_{i} \cap X_{j}\right)\right|}{|U|}+\ldots \\
& +(-1)^{n+1} \frac{\mid R_{A^{k}}^{* z}\left(X_{1}\right) \cap \frac{R_{A^{k}}^{* z}}{\left(X_{2}\right) \cap \ldots R_{A^{k}}^{* z}\left(X_{n}\right) \mid}}{|U|}
\end{aligned}
$$

Hence, $\mathrm{Bel}_{A^{k}}^{*>}(X)$ is a belief function. And $P l_{A^{k}}^{* \geq}(X)$ is also a plausibility function due to the duality of the belief and plausibility functions.

With Theorem 3, there exactly exists the corresponding belief structure of multi-granulation rough set and the consistency of belief structure in different levels of granulations from Theorem 2 can be derived as follows.

Proposition 3 Let $M G O I S^{* \geq}=\left(U, A T^{k}, f^{k}, V^{k}\right)$ be an incomplete multi-granulation ordered information system which has I levels of granulations. For any $1 \leq k \leq I$, denote the subset $A^{k} \subseteq A T^{k}$. For $x \subseteq U, \operatorname{Bel}_{A^{k}}^{* \geq}(X)$ and $P l_{A^{k}}^{* \geq}(X)$ are the belief and plausibility functions, respectively, and $P(X)=\frac{|X|}{|U|}$. By the above analysis, we have the properties as follows.

(1) $B e l_{A^{I}}^{* \geq}(X) \leq B e l_{A^{I-1}}^{* \geq}(X) \leq \ldots \leq B e l_{A^{1}}^{* \geq}(X) \leq P(X)$

(2) $P(X) \leq P l_{A^{1}}^{* \geq}(X) \leq P l_{A^{2}}^{* \geq}(X) \leq \ldots \leq P l_{A^{I}}^{* \geq}(X)$ 


\subsection{Reduction in incomplete multi-granulation ordered information system}

First, the positive region reduction, belief reduction and plausibility reduction are put forward in incomplete multi-granulation ordered information system.

Definition 9 Let $M G O I S^{*} \geq=\left(U, A T^{k}, f^{k}, V^{k}\right)$ be an incomplete multi-granulation ordered information system which has $I$ levels of granulations. For any $1 \leq k \leq I$, denote the subset $A^{k} \subseteq A T^{k}$, and the positive region, belief and plausibility reduction are developed in the $k$-th level of granulations of information system as follows.

(1) If there exists $U / R_{A^{k}}^{* \geq}=U / R_{A^{k}}^{* \geq}$, then assume that $A^{k}$ is a consistent set. Furthermore, if any true subset of $A^{k}$ is not a consistent set, then $A^{k}$ can be defined as the positive region reduction.

(2) If there exists $B e l_{A^{k}}^{* \geq}(X)=B e l_{A T^{k}}^{* \geq}(X)$, where for every $X \in U / R_{A^{k}}^{* \geq}$, then assume that $A^{k}$ is a belief consistent set. Furthermore, if any true subset of $A^{k}$ is not a belief consistent set, then $A^{k}$ can be defined as belief reduction.

(3) If there exists $P l_{A^{k}}^{* \geq}(X)=P l_{A T^{k}}^{* \geq}(X)$, where for every $X \in U / R_{A^{k}}^{* \geq}$, then assume that $A^{k}$ is a plausibility consistent set. Furthermore, if any true subset of $A^{k}$ is not a plausibility consistent set, then $A^{k}$ can be defined as plausibility reduction.

Based on Definition 9, belief reduction and plausibility reduction are the minimal attribute set to keep the degree of belief and plausibility. Next, we analyze the consistency of three ways of reduction in the same level of granulations.

Theorem 3 Let $M G O I S^{* \geq}=\left(U, A T^{k}, f^{k}, V^{k}\right)$ be an incomplete multi-granulation ordered information system which has I levels of granulations. For any $1 \leq k \leq I$, denote the subset $A^{k} \subseteq A T^{k}$, and we have some properties in the $k$-th level of granulations of information system as follows.

(1) If $A^{k}$ is the consistency set if and only if it is the belief consistency set.

(2) If $A^{k}$ is the positive region reduction if and only if it is the belief reduction.

(3) If $A^{k}$ is the consistency set if and only if it is the plausibility consistency set.

(4) If $A^{k}$ is the positive region reduction if and only if it is the plausibility reduction.

Proof

(1) Suppose that $A^{k}$ is the consistency set in the k-th level of granulations of information system. Then, we have $U / R_{A^{k}}^{* \geq}=U / R_{A^{k}}^{* \geq}$. Clearly, $A^{k}$ is also the belief consistency set in the same level with Definition 9. If $A^{k}$ is the belief reduction in the $k$-th level of granulations. Then, we have $\operatorname{Bel}_{A^{k}}^{* \geq}(X)=B e l_{A T^{k}}^{* \geq}(X), X \in U / R_{A^{k}}^{* \geq}$ (i). According to Eqs.(i), one obtains $B e l_{A^{k}}^{* \geq}\left([X]_{A T^{k}}^{* \geq}\right)=B e l_{A T^{k}}^{* \geq}\left([X]_{A T^{k}}^{* \geq}\right)$ (ii), According to Eqs.(ii), one obtains $\frac{\left|R_{A^{k}}^{* \geq}\left([X]_{A T^{k}}^{* \geq}\right)\right|}{|U|}=\frac{\left|R_{A T^{k}}^{* \geq}\left([X]_{A T^{k}}^{* \geq}\right)\right|}{|U|}$ (iii). According to the conjunction of Theorem 2 
and Eqs(iii), we can obtain $R_{A^{k}}^{* \geq}\left([X]_{A T^{k}}^{* \geq}\right)=R_{A T^{k}}^{* \geq}\left([X]_{A T^{k}}^{* \geq}\right)$. By the definition of the lower approximation from Definition 7 and $\overline{A^{k} \subseteq A} T^{k}$,we can get the following relationship: $\left\{y \mid[y]_{A^{k}}^{* \geq} \subseteq[X]_{A T^{k}}^{* \geq}\right\}=\left\{y \mid[y]_{A T^{k}}^{* \geq} \subseteq[X]_{A T^{k}}^{* \geq}\right\} \leftrightarrow[X]_{A^{k}}^{* \geq} \subseteq[X]_{A T^{k}}^{* \geq}, x \in U$. Since $[X]_{A T^{k}}^{* \geq} \subseteq[X]_{A^{k}}^{* \geq}$, thus $[X]_{A T^{k}}^{* \geq}=[X]_{A^{k}}^{* \geq}$. Consequently, $A^{k}$ is the consistency set in the $k$-th level of granulations of information system.

(2) Similar to the proof of (1), it can be proved.

(3) Suppose that $A^{k}$ is the consistency set in the $k$-th level of granulations of information system. Then, we have $U / R_{A^{k}}^{* \geq}=U / R_{A^{k}}^{* \geq}$. Clearly, $A^{k}$ is also the plausibility consistency set in the same level with Definition 9.

(4) Similar to the proof of (3), it can be proved. As demonstrated above, we obtain the consistency of several reduction in the same level, which means the positive region reduction is equivalent to belief reduction. And it is also proved that the positive region reduction and belief reduction are adequate condition for plausibility reduction. Next, analyze the transitivity of the above three reduction in different levels.

Theorem 4 Let $M G O I S^{*} \geq=\left(U, A T^{k}, f^{k}, V^{k}\right)$ be an incomplete multi-granulation ordered information system which has I levels of granulations. For any $1 \leq k \leq I$, denote the subset $A^{k} \subseteq A T^{k}$ and we have some properties as follows.

(1) For any $x \in U$, we can obtain $[X]_{A^{k}}^{* \geq}=[X]_{A^{k+1}}^{* \geq}$, where $A^{k}$ is the positive region reduction in the $k$-th level of granulations of information system. Then, we define that $A^{k+1}$ is the positive region reduction in the $k$-th level of granulations.

(2) Reversely, if for $x \in U$,we can obtain $[X]_{A^{k}}^{* \geq}=[X]_{A^{k+1}}^{* \geq}$, where $A^{k+1}$ is the positive region reduction in the $(k+1)$-th level of granulations of information system. Then, we define that $A^{k}$ is the positive region reduction in the $(k+1)$-th level of granulations.

(3) Conversely, iffor $x \in U$, we can obtain $[X]_{A^{k}}^{* \geq}=[X]_{A^{k+1}}^{* \geq}$, where $A^{k+1}$ is not the positive region reduction in the $(k+1)$-th level of granulations of information system. Then, we define that $A^{k}$ is not the positive region reduction in the $k$-th level of granulations.

(1) If $A^{k}$ is the positive region reduction in the $k$-th level of granulations of information system, then we get $[X]_{A^{k}}^{* \geq}=[X]_{A T^{k}}^{* \geq}$, where $\forall x \in U$. By $[X]_{A^{k}}^{* \geq} \subseteq[X]_{A T^{k}}^{* \geq}$, we can obtain $[X]_{A^{k+1}}^{* \geq} \subseteq[X]_{A T^{k}}^{* \geq}$. And then, $[X]_{A^{k+1}}^{* \geq}=[X]_{A T^{k}}^{* \geq}$ can be verified. Hence, we get the result that $A^{k+1}$ is the positive region reduction in the $k$-th level of granulations.

(2) Similar to the proof of (1), it can be proved.

(3) If $A^{k+1}$ is not the positive region reduction in the $(k+1)$-th level of granulations, then we get $[X]_{A T^{k+1}}^{* \geq} \subset[X]_{A^{k+1}}^{* \geq}$. 
By $[X]_{A T^{k}}^{* \geq} \subseteq[X]_{A T^{k+1}}^{* \geq}$, we can obtain $[X]_{A T^{k}}^{* \geq} \subset[X]_{A T^{k+1}}^{* \geq}$.

And then, by $[X]_{A^{k+1}}^{* \geq}=[X]_{A^{k}}^{* \geq},[X]_{A T^{k}}^{* \geq} \subset[X]_{A^{k}}^{* \geq}$ can be verified.

Hence, we can get the result that $A^{k}$ is not the positive region reduction in the $k$-th level of granulations.

It is demonstrated in Theorem 4 that, when the condition $[X]_{A^{k}}^{* \geq}=[X]_{A^{k}+1}^{*>}$ where for every $x \in U$ is satisfied, reducibility has bidirectional transitivity between different levels of granulations and nonreducibility has only unidirectional transitivity between different levels of granulations, i.e., if the higher level of granulations has nonreducibility, then the lower level has irreducibility, too. Whereas it is impossible to judge whether the higher level can be reduced if the lower level has irreducibility. On the above analysis, the following inference can be put forward.

(1) Suppose that $A^{i}$ is the positive region reduction in the $i$-th level of granulations and for every $x \in U$,we have $[X]_{A^{k}}^{* \geq}=[X]_{A^{k}+m}^{* \geq}$, where $m>0, k+m \leq I$ and for every $I$, there exists $k \leq i \leq k+m$. Then, $A^{k}, A^{k+1}, \ldots, A^{k+m}$ are the positive region reductions in the $k$-th, $(k+1)$-th, $\cdots,(k+m)$-th levels of granulations of information system, respectively. Specially, when $i=1$, incomplete multi-granulation ordered information system completely can be positively reduced.

(2) Conversely, suppose that $A^{i}$ is not the positive region reduction in the $i$-th level of granulations and for every $x \in U$, we have $[X]_{A^{k}}^{* \geq}=[X]_{A^{k}+m}^{* \geq}$, where $m>0$, $k+m \leq I$ and for every $I$, there exists $k \leq i \leq k+m$. Then, $A^{k}, A^{k+1}, \ldots, A^{k+m}$ are not the positive region reductions in the $k$-th, $(k+1)$-th, . ., $(k+m)$-th levels of granulations of information system, respectively. Specially, when $i=1$, incomplete multigranulation ordered information system completely can't be positive region reduced.

(1) First, prove that $A^{k}$ is the positive region reduction in the $i$-th level of granulations. If $A^{i}$ is the positive region reduction in the $i$-th level of granulations of information system, then we get $[X]_{A^{i}}^{* \geq} \subseteq[X]_{A T^{i}}^{* \geq}$, where $\forall x \in U$. For $k \leq i \leq k+m \leq I$ and $[X]_{A^{k}}^{* \geq}=[X]_{A^{k+m}}^{* \geq},[X]_{A^{k}}^{* \geq}=[X]_{A^{k+m}}^{* \geq}=[X]_{A^{i}}^{* \geq}$ can be obtained. By $[X]_{A^{i}}^{* \geq} \subseteq[X]_{A T^{i}}^{* \geq}$, we can obtain $[X]_{A^{k}}^{* \geq} \subseteq[X]_{A T^{i}}^{* \geq}$. And then, $[X]_{A^{k}}^{* \geq}=[X]_{A T^{i}}^{* \geq}$ can be verified. Thus, we get that $A^{k}$ is the positive region reduction in the $i$-th level of granulations. Second, similar to proof of (1), we can derive that $A^{i}$ is the positive region reduction in the $k$-th level of granulations, $A^{i}$ is the positive region reduction in the $(k+m)$-th level of granulations and $A^{k+m}$ is the positive region reduction in the $i$-th level of granulations Hence, we can get the result that $A^{k}, A^{k+1}, \ldots, A^{k+m}$ are the positive region reductions in the $k$-th, $(k+1)$-th, $\cdots,(k+m)$-th levels of granulations of information system, respectively.

(2) First, prove that $A^{k}$ is not the positive region reduction in the k-th level of granulations. 
If $A^{i}$ is the positive region reduction in the $i$-th level of granulations of information system, then we get $[X]_{A T^{i}}^{* \geq}=[X]_{A^{i}}^{* \geq}$, where $\forall x \in U$.

For $k \leq i \leq k+m \leq I$ and $[X]_{A^{k}}^{* \geq}=[X]_{A^{k+m}}^{* \geq},[X]_{A^{k}}^{* \geq}=[X]_{A^{k+m}}^{* \geq}=[X]_{A^{i}}^{* \geq}$ can be obtained.

By $[X]_{A T^{k}}^{* \geq} \subseteq[X]_{A T^{i}}^{* \geq}$, we can obtain $[X]_{A^{k}}^{* \geq} \subset[X]_{A T^{i}}^{* \geq}$.

And then, $[X]_{A T^{k}}^{* \geq} \subset[X]_{A^{k}}^{* \geq}$ can be verified.

Thus, we can get that $A^{k}$ is not the positive region reduction in the $k$-th level of granulations.

Second, similar to proof of (1), we can derive that $A^{k+m}$ is not the positive region reduction in the $(k+m)$-th level of granulations.

Hence, we can get the result that $A^{k}, A^{k+1}, \ldots, A^{k+m}$ are not the positive region reductions in the $k$-th, $(k+1)$-th, . , $(k+m)$-th levels of granulations of information system, respectively.

In order to describe the relationship among the reductions above conveniently, given the relationship figures as follows, clearly, Fig. 2 is the consistency of several reductions in the same level of granulations. In this representation, each of nodes represents a kind of reduction and the unidirectional arrows are granted as a reduction of the end point from the starting point, i.e., the positive region reduction $A$ must be the plausibility reduction at the mean time. The bidirectional arrows mean the equivalent of the ends of the arrow. Figure 3 represents the transitivity of several reductions in the different levels of granulations. When $[X]_{A^{k}}^{* \geq} \subset[X]_{A^{k+1}}^{* \geq}$ are satisfied, we can get that $A$ is also the positive region reduction of the $k$-th and $(k+1)$-th levels of granulations.

\section{Results and discussion}

In this section, we will simulate numerically the relationship of three reductions of an incomplete multi-granulation ordered information system based on belief structure that is defined in Sect. 3 and give the notions of the significance to explain whether the corresponding attribute is dispensable or not. Based on this fact, we proposed an algorithm to find out reductions of an incomplete multi-granulation ordered information system.

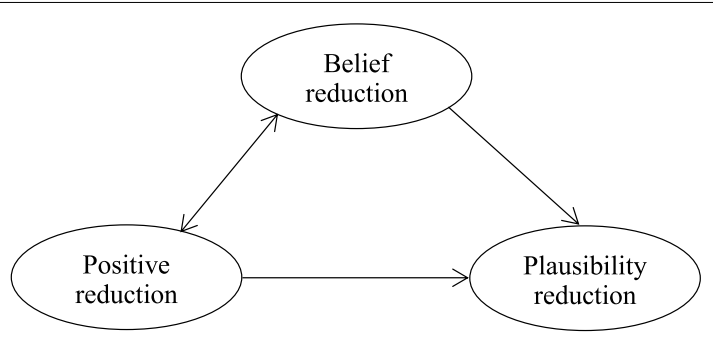

Fig. 2 The consistency of several reductions in the same levels of granulations 


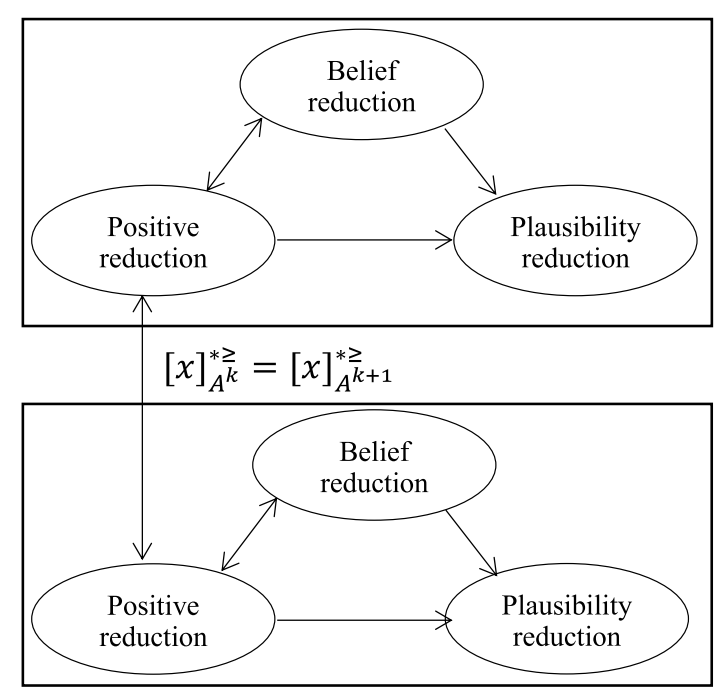

Fig. 3 The transitivity of several reductions between $k$ and $k+1$ levels of granulations

\subsection{Algorithm design}

Definition 10 Let $M G O I S^{* \geq}=\left(U, A T^{k}, f^{k}, V^{k}\right)$ be an incomplete multi-granulation ordered information system. If $A T^{k}=\left\{a_{1}^{k}, a_{2}^{k}, \ldots, a_{m}^{k}\right\}$, then for every attribute $a_{i}^{k} \in A T^{k}$, we define the significance degree of the attribute $A T^{k}$ as follows.

$$
\begin{aligned}
& \operatorname{sig}_{1}\left(a_{i}^{k}, A T^{k}\right)=\frac{U}{R_{A T^{k}}}-\frac{U}{R_{A T^{k}-\left\{a_{i}^{k}\right\}}} \\
& \operatorname{sig}_{2}\left(a_{i}^{k}, A T^{k}\right)=B e l_{A T^{k}}-B e l_{A T^{k}-\left\{a_{i}^{k}\right\}} \\
& \operatorname{sig}_{3}\left(a_{i}^{k}, A T^{k}\right)=P l_{A T^{k}}-P l_{A T^{k}-\left\{a_{i}^{k}\right\}}
\end{aligned}
$$

$\operatorname{sig}_{1}\left(a_{i}^{k}, A T^{k}\right)>0$ explains that the attribute $a_{i}^{k}$ is not dispensable for $A T^{k}$ and $a_{i}^{k}$ should be included in the positive region reduction. If $\operatorname{sig}_{1}\left(a_{i}^{k}, A T^{k}\right) \leq 0$, it shows the attribute $a_{i}^{k}$ is dispensable for $A T^{k}$ and $a_{i}^{k}$ should not be included in positive region reduction. $\operatorname{sig}_{2}\left(a_{i}^{k}, A T^{k}\right)>0$ explains that the belief function of the attribute $a_{A T-\left\{a_{i}^{k}\right\}}^{k}$ is equal to $A T^{k}$, which means $a_{i}^{k}$ should be included in the belief reduction. If $\operatorname{sig}_{2}\left(a_{i}^{k}, A T^{k}\right) \leq 0$, it shows the belief function of the attribute $a_{A T-\left\{a_{i}^{k}\right.}^{k}$ is not equal to $A T^{k}$, and $a_{i}^{k}$ should not be included in belief reduction. $\operatorname{sig}_{3}\left(a_{i}^{k}, A T^{k}\right)>0$ explains that the plausibility function of the attribute $a_{A T-\left\{a_{i}^{k}\right\}}$ is equal to $A T^{k}$ and $a_{i}^{k}$ should be included in the plausibility reduction. If $\operatorname{sig}_{3}\left(a_{i}^{k}, A T^{k}\right) \leq 0$, it shows the plausibility function of the attribute $a_{A T-\left\{a_{i}^{k}\right\}}^{k}$ is equal to $A T^{k}$, and $a_{i}^{k}$ should not be included in the plausibility reduction. 
The specific steps of the reduction will be given in the following. Since that it is same for reduction steps in different levels of granulations, just given the reduction process of a certain level of granulations as Algorithm 1 shows.

Algorithm 1 Reduction in incomplete multi-granulation ordered information system based on belief structure

Input: incomplete information system $I S^{* \geq}=(U, A T, f, V)$;

Output: let the positive region reduction be $R e d$, belief reduction be $\operatorname{Re} d_{B e l}$, plausibility reduction be $\operatorname{Red}_{P l}$.

Step 1: let $\operatorname{Red}=\varnothing, \operatorname{Red}_{B e l}=\varnothing, \operatorname{Red} P l=\varnothing$ and $A T^{\prime}=A T$;

Step 2: according to Definition 9, calculate the positive region consistent set $U / R_{A T}$, belief consistent set $B e l_{A T}$ and plausibility consistent set $P l_{A T}$ of the attribute $A T$;

Step 3: let $a_{i} \in A T$, according to Definition 9, calculate the positive region consistent set $U / R_{A T-\left\{a_{i}\right\}}$, belief consistent set $B e l_{A T-\left\{a_{i}\right\}}$ and plausibility consistent set $P l_{A T-\left\{a_{i}\right\}}$ of the attribute $A T-\left\{a_{i}\right\}$

Step 4: Let $\operatorname{sig}_{j}\left(a_{i}, A T\right)$ be the significance and relative of the attribute $a_{i}$ wherel $\leq|j| \leq 3$, $\operatorname{sig}_{1}\left(a_{i}, A T\right)=U / R_{A T^{k}}-U / R_{A T-\left\{a_{i}\right\}}, \quad \operatorname{sig}_{2}\left(a_{i}, A T\right)=\operatorname{Bel}_{A T}-\operatorname{Bel}_{A T-\left\{a_{i}\right\}} \quad$ and $\operatorname{sig}_{3}\left(a_{i}, A T\right)=P l_{A T}-P l_{A T-\left\{a_{i}\right\}}$

Step 5: If $\operatorname{sig}_{1}\left(a_{i}, A T\right)>0$, then suppose the attribute $a_{i}$ is important, and add it into the positive region reduction set, and obtain $a_{i} \in \operatorname{Red}$, then go to Step 8 , else go to Step 8 directly;

Step 6: If $\operatorname{sig}_{2}\left(a_{i}, A T\right)>0$, then suppose the attribute $a_{i}$ is important, and add it into the belief reduction set, and obtain $a_{i} \in \operatorname{Red}_{B e l}$, then go to Step 8, else go to Step 8 directly;

Step 7: If $\operatorname{sig}_{3}\left(a_{i}, A T\right)>0$, then suppose the attribute $a_{i}$ is important, and add it into the plausibility reduction set, and obtain $a_{i} \in \operatorname{Red}_{P l}$, then go to Step 8 , else go to Step 8 directly;

Step 8: Let $A T^{\prime}=A T^{\prime}-\left\{a_{i}\right\}$, if $A T^{\prime}=\varnothing$, then return step 8, else let $a_{i}=a_{j}$, and return Step 3, where $i \neq j$;

Step 9: output the positive region reduction $R e d$, belief reduction $\operatorname{Red}_{B e l}$ and plausibility reduction $\operatorname{Red} P l$ as reduction.

Suppose that the size of $U$ is $\mathrm{n}$ and the number of attributions is $m$, then the time complexity of Algorithm 1 is $O(m * n)$. Table 5 and Fig. 4 both show the comparison of time complexity among different rough set measures, like covering rough set [25], traditional 
Table 5 The comparison of time complexity of different rough set measures

\begin{tabular}{lllll}
\hline Measure & MGRS based on belief structure & MGRS & Covering rough set & Traditional rough set \\
\hline Time complexity & $O(m * n)$ & $O\left(n^{2}\right)$ & $O\left(m^{2} * n^{2}\right)$ & $O\left(m * n^{2}\right)$ \\
\hline
\end{tabular}

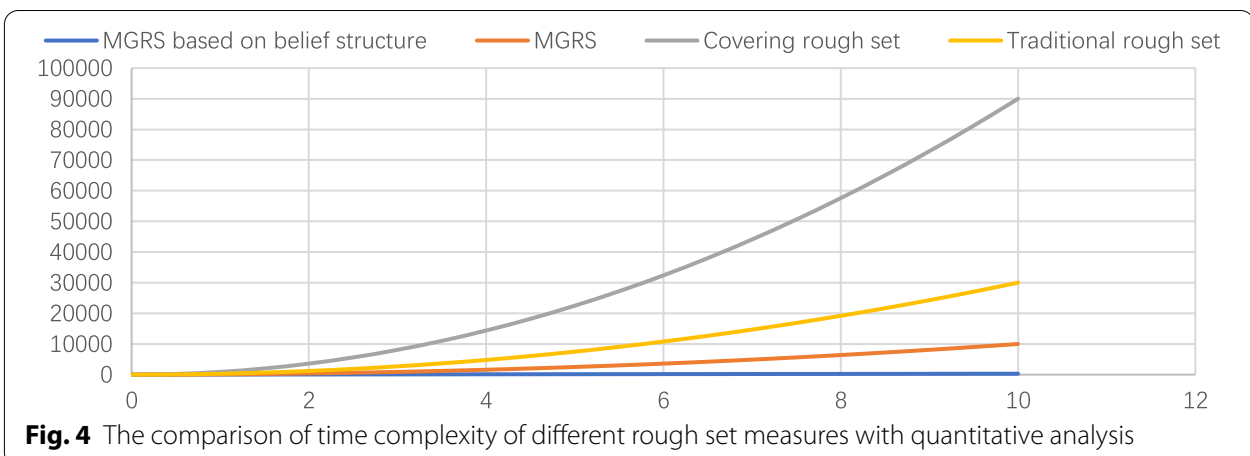

rough set [7] and multi-granulation rough set [18], illustrating that the calculating time can be reduced largely. In Fig. 4 , suppose $m=30$ and $n=0,500,1000, \ldots, 10000$. For clearly observing, the final result of our idea is divided by 1000, MGRS is divided by 10000 , and the rest methods are divided by 100000 . This figure shows the great superiority of MGRS based on belief structure with low time complexity clearly and intuitively. And the following analysis of Example 1, which is analyzed briefly in Sect. 3.2, is employed to illustrate our idea.

\subsection{Algorithm implementation}

Example 1.1. It is an incomplete multi-granulation ordered information system table of Rape pests detected by WSN in a certain period of time, where granularity $I=3$, $U=\left\{x_{1}, x_{2}, x_{3}, x_{4}, x_{5}, x_{6}, x_{7}, x_{8}, x_{9}, x_{1} 0, x_{1} 1, x_{1} 2\right\} \quad$ and $\quad A T^{k}=\left\{a_{1}^{k}, a_{2}^{k}, a_{3}^{k}, a_{4}^{k}\right\}$. $X_{i}(i=1,2, \ldots, 12)$ represents different clusters which stand for Cabbage butterfly, Aphids, Cabbage bug and Cricket, respectively. And $A T^{k}$ is the attribute set where $k=1,2,3$, which is the different levels of granulations. Since that, it is same for reduction steps in different levels of granulations, just given the reduction process of the first level of granulations as Algorithm 1 shows.

(1) Let $\operatorname{Red}=\varnothing, \operatorname{Red}_{B e l}=\varnothing, \operatorname{Red}_{P l}=\varnothing$ and $A T^{\prime}=A T$;

(2) According to Definition 9, calculate the positive region consistent set $U / R_{A T^{1}}$, belief consistent set $B e l_{A T^{1}}$ and plausibility consistent set $P l_{A T^{1}}$ of the attribute $A T^{1}$.

$$
U / R_{A T^{1}}=\left\{a_{1}^{1}, a_{2}^{1}, a_{3}^{1}, a_{4}^{1}\right\}, B e l_{A T^{1}}=\left\{a_{1}^{1}, a_{2}^{1}, a_{3}^{1}, a_{4}^{1}\right\}, P l_{A T^{1}}=\left\{a_{1}^{1}, a_{2}^{1}, a_{3}^{1}, a_{4}^{1}\right\}
$$


(3) Calculate the positive region consistent set $U / R_{A T^{1}}=U / R_{A T^{1}-\left\{a_{1}^{1}\right\}}$, belief consistent set $B e l_{A T^{1}-\left\{a_{1}^{1}\right\}}$ and plausibility consistent set $P l_{A T^{1}-\left\{a_{1}^{1}\right\}}$ of the attribute $A T^{1}-\left\{a_{1}^{1}\right\}$. And we can get $[x]_{A T^{1}-\left\{a_{1}^{1}\right\}}^{* \geq}$ from Table 2 as follows.

$$
\begin{aligned}
& {\left[x_{1}\right]_{A T^{1}-\left\{a_{1}^{1}\right\}}^{* \geq}=\left[x_{2}\right]_{A T^{1}-\left\{a_{1}^{1}\right\}}^{* \geq}=\left\{x_{1}, x_{2}, x_{8}, x_{10}\right\}} \\
& {\left[x_{3}\right]_{A T^{1}-\left\{a_{1}^{1}\right\}}^{* \geq}=\left[x_{7}\right]_{A T^{1}-\left\{a_{1}^{1}\right\}}^{* \geq}=\left[x_{12}\right]_{A T^{1}-\left\{a_{1}^{1}\right\}}^{* \geq}=\{U\}} \\
& {\left[x_{4}\right]_{A T^{1}-\left\{a_{1}^{1}\right\}}^{* \geq}=\left[x_{9}\right]_{A T^{1}-\left\{a_{1}^{1}\right\}}^{* \geq}=\left\{x_{1}, x_{2}, x_{4}, x_{6}, x_{8}, x_{9}, x_{10}, x_{11}\right\}} \\
& {\left[x_{5}\right]_{A T^{1}-\left\{a_{1}^{1}\right\}}^{* \geq}=\left\{x_{1}, x_{2}, x_{4}, x_{5}, x_{6}, x_{8}, x_{9}, x_{10}, x_{11}\right\}} \\
& {\left[x_{6}\right]_{A T^{1}-\left\{a_{1}^{1}\right\}}^{* \geq}=\left\{x_{1}, x_{2}, x_{6}, x_{8}, x_{10}\right\}} \\
& {\left[x_{8}\right]_{A T^{1}-\left\{a_{1}^{1}\right\}}^{* \geq}=\left[x_{10}\right]_{A T^{1}-\left\{a_{1}^{1}\right\}}^{* \geq}=\left\{x_{1}, x_{8}, x_{10}\right\}} \\
& {\left[x_{11}\right]_{A T^{1}-\left\{a_{1}^{1}\right\}}^{* \geq}=\left\{x_{1}, x_{2}, x_{6}, x_{8}, x_{10}, x_{11}\right\}}
\end{aligned}
$$

Thus, $U / R_{A T^{1}-\left\{a_{1}^{1}\right\}}=\left\{a_{1}^{1}, a_{2}^{1}, a_{3}^{1}, a_{4}^{1}\right\}$. For every $X \in U / R_{A T^{k}}^{* \geq}$, suppose $X=\left\{x_{1}, x_{6}, x_{8}, x_{10}, x_{11}\right\}$, and the reduction of the lower and upper approximation of $A T^{1}-\left\{a_{1}^{1}\right\}$ are $\underline{R_{A T^{1}-\left\{a_{1}^{1}\right\}}^{* \geq}}(X)=\left\{x_{8}, x_{10}\right\}$ and $\overline{R_{A T^{1}-\left\{a_{1}^{1}\right\}}^{* \geq}}(X)=\{U\}$, respectively. Additionally, the reduction of the lower and upper approximation of $\underline{R_{A T^{1}}^{* \geq}}(X)=\left\{x_{1}, x_{8}, x_{10}\right\} \quad$ and $\quad \overline{R_{A T^{1}}^{* \geq}}(X)=\{U\}, \quad$ respectively. Hence,

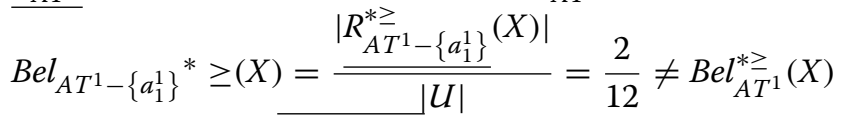

$P l_{A T^{1}-\left\{a_{1}^{1}\right\}}^{* \geq}(X)=\frac{\left|\overline{R_{A T^{1}-\left\{a_{1}^{1}\right\}}^{* \geq}}(X)\right|}{|U|}=1=P l_{A T^{1}}^{* \geq}(X)$.

Consequently, $\operatorname{Bel}_{A T^{1}}=\left\{a_{1}^{1}, a_{2}^{1}, a_{3}^{1}, a_{4}^{1}\right\}, P l_{A T^{1}}=\left\{a_{2}^{1}, a_{3}^{1}, a_{4}^{1}\right\}$.

(4) Thus, we get $\operatorname{sig}_{1}\left(a_{1}^{1}, A T^{1}\right)=U / R_{A T^{1}}-U / R_{A T^{1}-\left\{a_{1}^{1}\right\}}=\{\varnothing\}=0$, $\operatorname{sig}_{2}\left(a_{1}^{1}, A T^{1}\right)=\operatorname{Bel}_{A T^{1}}-\operatorname{Bel}_{A T^{1}-\left\{a_{1}^{1}\right\}}=\{\varnothing\}=0$ and $\operatorname{sig}_{3}\left(a_{1}^{1}, A T^{1}\right)=P l_{A T^{1}}-P l_{A T^{1}-\left\{a_{1}^{1}\right\}}=\left\{a_{1}^{1}\right\}>0$. Then, add the attribute $a_{1}$ into plausibility reduction $\operatorname{Red}_{P l}$.

(5) Similarly, $\quad B e l_{A T^{1}-\left\{a_{2}^{1}\right\}}^{* \geq}(X)=3 / 12$, $P l_{A T^{1}-\left\{a_{2}^{1}\right\}}^{*} \geq(X)=1$, $\operatorname{sig}_{1}\left(a_{2}^{1}, A T^{1}\right)=U / R_{A T^{1}}-U / R_{A T^{1}-\left\{a_{2}^{1}\right\}}=\left\{a_{2}^{1}\right\}>0$ $\operatorname{sig}_{2}\left(a_{2}^{1}, A T^{1}\right)=\operatorname{Bel}_{A T^{1}}-\operatorname{Bel}_{A T^{1}-\left\{a_{2}^{1}\right\}}=\left\{a_{2}^{1}\right\}>0$ and $\operatorname{sig}_{3}\left(a_{2}^{1}, A T^{1}\right)=P l_{A T^{1}}-P l_{A T^{1}-\left\{a_{2}^{1}\right\}}=\left\{a_{2}^{1}\right\}>0$, then add $a_{2}$ into Red, Red $\operatorname{Rel}_{B e}$, $\operatorname{Red}_{P l} . \quad \quad \operatorname{Bel}_{A T^{1}-\left\{a_{3}^{1}\right\}}^{* \geq}(X)=1 / 12, \quad \quad P l_{A T^{1}-\left\{a_{3}^{1}\right\}}^{* \geq}(X)=1$, $\operatorname{sig}_{1}\left(a_{3}^{1}, A T^{1}\right)=U / R_{A T^{1}}-U / R_{A T^{1}-\left\{a_{3}^{1}\right\}}=\{\varnothing\}=0$ 


$$
\begin{aligned}
& \operatorname{sig}_{2}\left(a_{3}^{1}, A T^{1}\right)=B e l_{A T^{1}}-\operatorname{Bel}_{A T^{1}-\left\{a_{3}^{1}\right\}}=\{\varnothing\}=0 \\
& \operatorname{sig}_{3}\left(a_{3}^{1}, A T^{1}\right)=P l_{A T^{1}}-P l_{A T^{1}-\left\{a_{3}^{1}\right\}}=\left\{a_{3}^{1}\right\}>0, \quad \text { then } \quad \text { add } a_{3} \quad \text { into } \operatorname{Red}_{P l} . \\
& \operatorname{Bel}_{A T^{1}-\left\{a_{4}^{1}\right\}}^{* \geq}(X)=3 / 12, \\
& \operatorname{sig}_{1}\left(a_{4}^{1}, A T^{1}\right)=U / R_{A T^{1}}-U / R_{A T^{1}-\left\{a_{4}^{1}\right\}}=\left\{a_{4}^{1}\right\}>0 \\
& \operatorname{sig}_{2}\left(a_{4}^{1}, A T^{1}-\left\{a_{4}^{1}\right\}\right. \\
& \operatorname{sig}_{3}\left(a_{4}^{1}, A T^{1}\right)=\operatorname{Bel}_{A T^{1}}-\operatorname{Bel}_{A T^{1}-\left\{a_{4}^{1}\right\}}=\left\{a_{4}^{1}\right\}>0 \\
& \operatorname{Red}_{P l} .
\end{aligned}
$$

From the analysis of Table 1 from Example 1, we can obtain that $A^{1}=\left\{a_{2}^{1}, a_{4}^{1}\right\}$ is the reduction of $A T^{1}$ in the first level of granulations and $A^{2}=\left\{a_{2}^{2}, a_{4}^{2}\right\}$ is the reduction of $A T^{2}$ in the second level of granulations where for every $x \in U$, there exists $[X]_{A^{1}}^{* \geq}=[X]_{A^{2}}^{* \geq}$. However, $A^{3}=\left\{a_{2}^{3}, a_{4}^{3}\right\}$ is not the reduction of $A T^{3}$ in the third level of granulations. This result does prove the idea that the positive and belief reduction are equivalent, and these two reductions are unnecessary and sufficient condition for plausibility reduction in the same level, and if cover structure order of different levels are the same the corresponding equivalent positive region reduction. In this example, data redundancy is reduced by half by attribute reduction based on belief structure, which largely improves the information processing capability of sensor nodes.

\section{Conclusion}

Considering the issue with respect to the high data redundancy and high cost of information collection in wireless sensor nodes, this paper proposes a data fusion method based on belief structure to reduce attribution in multi-granulation rough set. In this paper, the relationship between multi-granulation rough set based on multi-scale and evidence theory is explored to analyze that the former has its corresponding belief structure when certain conditions are satisfied. Then, by introducing evidence structure, the positive region, belief and plausibility reduction are put forward and analyze the consistency in the same level and transitivity in the different levels by means of information system. Meanwhile, the algorithm proposed in this paper to perform three reductions reduces the time complexity largely, improving the computing efficiency. In essence, this paper not only successfully solves the problem of sensor node data fusion, but also provides new ideas and methods for the research in incomplete multi-granulation ordered rough set theory.

However, one of the drawbacks of the proposed algorithm is that it requires attribute discretization and it will lose efficacy when the reducible attributes are few. And the future work will concentrate on the synthesis of multi-granulation ordered information system evidence under the belief space with respect to data fusion in wireless sensor networks.

\section{Abbreviations}

WSN: Wireless sensor network; Eqs: Equally spaced. 


\section{Authors' contributions}

Chenfeng Long is main author of the current paper. Chenfeng Long contributed to the development of the idea, design of the study, theory, result analysis, and article writing. Xingxin Liu was responsible for the reducing attribute, processing the algorithm and drawing figures. Yakun Yang and Tao Zhang were responsible for collecting data. Siqiao Tan participated in the design of the study. Kui Fang have suggested contributed amendments. Xiaoyong Tang helped to draft the manuscript. Gelan Yang participated in the result analysis. All authors read and approved the final manuscript.

\section{Funding}

This research was partially funded by the National Natural Science Foundation of China (Grant No. 61972146, 61672219)

\section{Availability of data and materials}

The datasets used and/or analyzed during the current study are available from the corresponding author on reasonable request.

\section{Declarations}

Ethics approval and consent to participate

This article does not contain any studies with human participants or animals performed by any of the authors.

\section{Consent for publication}

All authors agree to submit this version and claim that no part of this manuscript has been published or submitted elsewhere.

\section{Competing interests}

The authors declare that they have no competing interests.

\section{Author details}

${ }^{1}$ School of Information and Intelligence Science and Technology, Hunan Agricultural University, Changsha, China.

${ }^{2}$ School of Computer and Communications Engineering, Changsha University of Science and Technology, Changsha,

China. ${ }^{3}$ Department of Computer Science, Hunan City University, Yiyang, China.

Received: 19 October 2020 Accepted: 1 February 2021

Published online: 08 April 2021

\section{References}

1. D. Zhifeng, L. Yuanxiang, L. Feng, W. Weiwu, Hierarchical intelligent method of information processing in wireless sensor networks. Comput. Eng. 09, 58-59(1996)

2. D. Zhifeng, L. Yuanxiang, Y. Li, X. Baiyi, Research on intelligent information processing in wireless sensor networks using rough set theory. Appl. Res. Comput. 10, 75-78 (2007)

3. X. Luo, J. Sun, L. Wang, W. Wang, W. Zhao, J. Wu, J. Wang, Z. Zhang, Short-term wind speed forecasting via stacked extreme learning machine with generalized correntropy. IEEE Trans. Ind. Inf. 14(11), 4963-4971 (2018)

4. Y. Zeng, C.J. Sreenan, N. Xiong, L.T. Yang, J.H. Park, Connectivity and coverage maintenance in wireless sensor networks. J. Supercomput. 52(1), 23-46 (2010)

5. W. Guo, N. Xiong, H.C. Chao, S. Hussain, G. Chen, Design and analysis of self-adapted task scheduling strategies in wireless sensor networks. Sensors. 11(7), 6533-6554 (2011)

6. H. Zheng, W. Guo, N. Xiong, A kernel-based compressive sensing approach for mobile data gathering in wireless sensor network systems. IEEE Trans. Syst. Man Cybernet. 48(12), 2315-2327 (2018)

7. Z. Pawlak, Rough sets. Int. J. Comput. Inf. Sci. 11(5), 341-354 (1982)

8. Z.D.Z.I.S.L.A.W. PAWLAK, Rough set theory and its applications to data analysis. Cybernet. Syst. 29(7), 661-688 (1998)

9. P. Dan, Z. Qilun, Self-optimization algorithm for attribute reduction. Comput. Res. Dev. 38(8), 904-910 (2001)

10. F. Ang, W. Guoyin, H. Jun, Attribute reduction algorithm for incomplete information system based on information entropy. J. Chongqing Univ. Posts Telecommun. Nat. Sci. Ed. 20(5), 586-592 (2008)

11. Parvanov P. Angelov, Handbook on computational intelligence (in 2 volumes) granular computing, pp. 43-66 (2016)https://doi.org/10.1142/9548

12. H. Liu, A. Gegov, M. Cocea, Rule-based systems: a granular computing perspective. Granular Comput. 1(4), 259-274 (2016)

13. G. Wilke, E. Portmann, Granular computing as a basis of human-data interaction: a cognitive cities use case. Granular Comput. 1(3), 181-197 (2016)

14. A. Skowron, A. Jankowski, S. Dutta, Interactive granular computing. Granular Comput. 1(2), 95-113 (2016)

15. S.S. Kumar, H.H. Inbarani, Optimistic multi-granulation rough set based classification for medical diagnosis. Procedia Comput. Sci. 47, 374-382 (2015)

16. J.M. Ma, Y. Yao, Rough set approximations in multi-granulation fuzzy approximation spaces. Fundamenta Informaticae 142(1-4), 145-160 (2015)

17. Yiyu Yao, A triarchic theory of granular computing. Granular Comput. 1(2), 145-157 (2016)

18. W.Z. Wu, Y. Leung, Theory and applications of granular labelled partitions in multi-scale decision tables. Inf. Sci. 181(18), 3878-3897 (2011)

19. Y. Qian, J. Liang, Y. Yao, C. Dang, MGRS: a multi-granulation rough set. Inf. Sci. Int. J. 180(6), 949-970 (2010)

20. C. Dang, Y. Qian, J. Liang, MGRS in Incomplete Information Systems, 2013 IEEE International Conference on Granular Computing (GrC), San Jose, California, 2007. https://doi.org/10.1109/GrC.2007.10 
21. P.P. Shenoy, G. Shafer, Axioms for probability and belief-function propagation. Mach. Intell. Pattern Recognit. 9 , 169-198 (1990)

22. J. Inglis, A mathematical theory of evidence. Technometrics 20, 106 (1978)

23. S. Glenn, A Mathematical Theory of Evidence (Princeton University Press, Princeton, 1900).

24. Y. Yao, P. Lingras, Interpretations of belief functions in the theory of rough sets. Inf. Sci. 104(1-2), 81-106 (1998)

25. D. Chen, X. Zhang, W. Li, On measurements of covering rough sets based on granules and evidence theory. Inf. Sci. 317, 329-348 (2015)

26. D. Chen, W. Li, X. Zhang, S. Kwong, Evidence-theory-based numerical algorithms of attribute reduction with neighborhood-covering rough sets. Int. J. Approx. Reason. 55(3), 908-923 (2014)

27. G. Lin, J. Liang, Y. Qian, An information fusion approach by combining multigranulation rough sets and evidence theory. Inf. Sci. 314, 184-199 (2015)

28. L. Liu, G. Luo, K. Qin, X. Zhang, An algorithm based on logistic regression with data fusion in wireless sensor networks. Eurasip J. Wirele. Commun. Netw. 2017(1), 10 (2017)

29. W. Guo, N. Xiong, A.V. Vasilakos, G. Chen, H. Cheng, Multi-source temporal data aggregation in wireless sensor networks. Wirel. Pers. Commun. 56(3), 359-370 (2011)

30. C. Lin, Y. He, N. Xiong, An energy-efficient dynamic power management in wireless sensor networks (2006)

\section{Publisher's Note}

Springer Nature remains neutral with regard to jurisdictional claims in published maps and institutional affiliations.

\section{Submit your manuscript to a SpringerOpen ${ }^{\circ}$ journal and benefit from:}

- Convenient online submission

- Rigorous peer review

- Open access: articles freely available online

- High visibility within the field

Retaining the copyright to your article

Submit your next manuscript at $>$ springeropen.com 